\title{
Needle tract seeding following endoscopic ultrasound-guided fine-needle aspiration for pancreatic cancer: a report of two cases
}

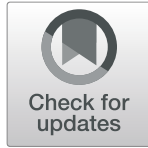

Toshiki Matsui $^{1 *}$, Kenichiro Nishikawa², Hiroki Yukimoto ${ }^{2}$, Koji Katsuta $^{3}$, Yoshihumi Nakamura², Shota Tanaka², Michiaki Oiwa ${ }^{2}$, Hiroki Nakahashi ${ }^{1}$, Yuta Shomi ${ }^{1}$, Yuji Haruki ${ }^{1}$, Kentaro Taniguchi ${ }^{1}$, Makoto Shimomura ${ }^{1}$ and Shuji Isaji ${ }^{4}$

\begin{abstract}
Background: Endoscopic ultrasound-guided fine-needle aspiration (EUS-FNA) is a useful tool in pancreatic cancer diagnosis. However, the procedure itself may cause peritoneal dissemination and needle tract seeding at the puncture site. We herein report two cases of gastric wall metastasis due to needle tract seeding after EUS-FNA.

Case presentation: Case 1: A 68-year-old woman was admitted to our hospital for persistent cough. Computed tomography (CT) scan revealed inflammatory changes in the left lung field, and incidentally, a 15-mm hypovascular mass was detected in the pancreatic body. She underwent EUS-FNA and was diagnosed as pancreatic adenocarcinoma. She underwent distal pancreatectomy with splenectomy; however, a small hard mass was observed in the posterior gastric wall during surgery. We performed partial gastrectomy, and the resected specimen was diagnosed as a needle tract seeding following EUS-FNA. She then underwent adjuvant chemotherapy with TS1 , but the pancreatic cancer showed recurrence 6 months after surgery. She died due to peritoneal dissemination 18 months after surgery.

Case 2: A 70-year-old man was incidentally detected with a pancreatic body mass on a CT scan as part of his follow-up for recurrence of basal cell carcinoma. He underwent EUS-FNA and was diagnosed as pancreatic adenocarcinoma. He had nodules in both lungs, and it was difficult to differentiate them from lung metastasis of pancreatic cancer. Therefore, he underwent neoadjuvant chemoradiotherapy, and thereafter, the lung nodules showed no changes; hence, he underwent distal pancreatectomy with splenectomy. During surgery, we observed a hard mass in the posterior gastric wall. We performed partial gastrectomy, and the resected specimen was diagnosed as needle tract seeding due to EUS-FNA. He underwent chemotherapy with TS-1, and he is still alive 18 months after surgery at the time of writing.
\end{abstract}

Conclusion: For resectable pancreatic body or tail tumors, EUS-FNA should be carefully performed to prevent needle tract seeding and intraoperative as well as postoperative assessment for gastric wall metastasis is mandatory.

Keywords: Endoscopic ultrasound-guided fine-needle aspiration, Needle tract seeding, Pancreatic cancer, Surgical resection, Gastric wall metastasis

\footnotetext{
*Correspondence: urinosuke0929@yahoo.co.jp

'Department of Surgery, Matsusaka Municipal Hospital, 1550, Tonomachi,

Matsusaka, Mie, Japan

Full list of author information is available at the end of the article
}

(c) The Author(s). 2019 Open Access This article is distributed under the terms of the Creative Commons Attribution 4.0 International License (http://creativecommons.org/licenses/by/4.0/), which permits unrestricted use, distribution, and reproduction in any medium, provided you give appropriate credit to the original author(s) and the source, provide a link to the Creative Commons license, and indicate if changes were made. The Creative Commons Public Domain Dedication waiver (http://creativecommons.org/publicdomain/zero/1.0/) applies to the data made available in this article, unless otherwise stated. 


\section{Background}

Endoscopic ultrasound-guided fine-needle aspiration (EUS-FNA) for pancreatic tumors has pooled sensitivity and specificity at $92 \%$ and $96 \%$, respectively [1], and it is an indispensable procedure for pancreatic cancer diagnosis. The main complications associated with EUSFNA for pancreatic tumors is bleeding, pancreatitis, and post-procedural pain, among others, but the incidence rate is as low as 1.03\%; therefore, EUS-FNA is considered a safe procedure [2]. The incidence rate of peritoneal dissemination associated with puncture for pancreatic cancer was reported to be $16.3 \%$ after percutaneous puncture and $2.2 \%$ after EUS-FNA; the risk of peritoneal dissemination is lower in EUS-FNA than in percutaneous puncture [3].

Needle tract seeding is a phenomenon in which tumor cells are found in the puncture route, and it is considered a subtype of peritoneal dissemination recurrence [4]. To the best of our knowledge, however, only 18 cases (17 reports) of needle tract seeding associated with EUS-FNA for pancreatic cancer have been reported till date [4-20]. Therefore, it is necessary to accumulate a greater number of cases of needle tract seeding for a better understanding of the features. Herein, we reported two cases of needle tract seeding after EUS-FNA that were detected during surgery and diagnosed via partial gastrectomy.

\section{Case presentation}

\section{Case 1}

A 68-year-old woman with no relevant medical or family history was admitted to our hospital because of a persistent cough. On admission, her abdomen was not tender and no mass was detected. Computed tomography (CT) scan revealed inflammatory signs in the left lung field along with incidental inflammatory findings around the pancreas, because of which pancreatitis was suspected. Dynamic-enhanced CT revealed a 15-mm hypovascular tumor in the pancreatic body (Fig. 1a), and inflammatory findings around the pancreas lead to the suspicion that concomitant pancreatitis is associated with pancreatic cancer. Laboratory data showed elevation of tumor marker levels (CA19-9, 44 U/ml; DUPAN-2, $1300 \mathrm{U} / \mathrm{ml}$; Span-1, 33.0 U/ml). Diffusion-weighted magnetic resonance image revealed high-signal intensity in pancreatic body tumor (Fig. 1b). Endoscopic ultrasonography (EUS) revealed a $14.7 \times 8.5 \mathrm{~mm}$ hypoechoic tumor in the pancreatic body, and the tumor did not contact to the superior mesenteric artery (SMA) and portal vein (PV). EUS-FNA for the pancreatic tumor was performed (4 punctures using $22 \mathrm{G}, 19 \mathrm{G}, 20 \mathrm{G}$, and $20 \mathrm{G}$ needles) via the trans-gastric approach, and no complications were noted (Fig. 1c). Cytology revealed adenocarcinoma (Fig. 1d). Based on the imaging findings, she was

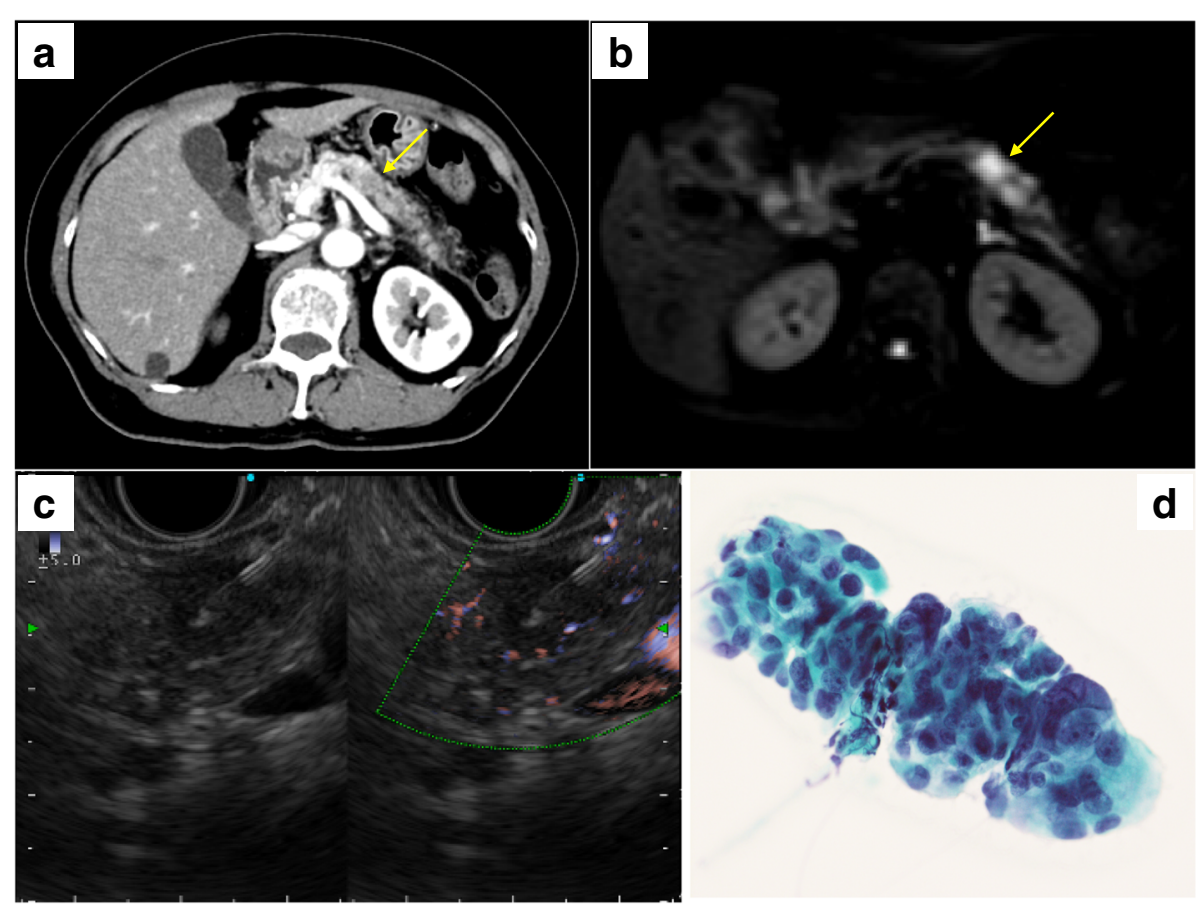

Fig. 1 a Dynamic-enhanced computed tomography (portal phase) for case 1. A 15-mm hypovascular tumor was detected in the pancreatic body (arrow). b Diffusion-weighted magnetic resonance imaging. A hyperintense area can be observed in the pancreatic body tumor (arrow). c Endoscopic ultrasound-guided fine-needle aspiration (EUS-FNA). EUS-FNA was performed for the pancreatic tumor (4 punctures using 22 G, 19 G, $20 \mathrm{G}$, and $20 \mathrm{G}$ needles) via the trans-gastric approach, without any complications. d Pathological findings of EUS-FNA. An adenocarcinoma can be observed (Papanicolaou staining) 
diagnosed as resectable pancreatic body cancer. She underwent distal pancreatectomy with splenectomy. However, during surgery, we noticed a small hard mass in the posterior gastric wall (Fig. 2a), and thus, we performed partial gastrectomy (Fig. 2b). The pathological findings of the specimen from partially resected stomach revealed adenocarcinoma cells which were linearly distributed in the gastric muscle layer; these findings indicated that the gastric tumor was needle tract seeding from pancreatic cancer due to EUS-FNA (Fig. 2c, d). The time from EUSFNA to the detection of the gastric wall metastasis due to needle tract seeding was 25 days. The pathological findings of the main pancreatic tumor resulted in a diagnosis of invasive ductal carcinoma, pT1, pN1 (No.8a, 11p), and pM0 pStageIIB (UICC). She then underwent adjuvant chemotherapy with TS-1, but a CT scan revealed peritoneal dissemination after 6 months. Therefore, the chemotherapy regimen was changed from TS-1 to gemcitabine (GEM) + nab-paclitaxel; however, her condition was gradually worsened and she died due to peritoneal dissemination of pancreatic cancer 18 months after surgery.

\section{Case2}

A 70-year-old man underwent CT scan as part of his follow-up for recurrence of basal cell carcinoma. Dynamic- enhanced CT scan incidentally revealed a $15-\mathrm{mm}$ hypovascular mass $15 \mathrm{~mm}$ in size in the pancreatic body (Fig. 3a). $\mathrm{He}$ had no abdominal symptoms, and laboratory data showed no elevation in tumor marker levels. Positron emission tomography-CT (PET-CT) revealed abnormal accumulation of fluorine-18- deoxyglucose (FDG) in the pancreatic body, with a standardized uptake value of 3.74 (Fig. 3b); however, there was no abnormal accumulation of FDG in other parts of the body. EUS revealed a 15.2-mm hypoechoic tumor in the pancreatic body. Although his tumor was suspected to invade the splenic artery, the tumor did not invade the SMA or PV. EUS-FNA was performed (1 puncture using a $22 \mathrm{G}$ needle) via the trans-gastric approach, and no complications occurred (Fig. 3c). Cytology revealed adenocarcinoma (Fig. 3d). He had small nodules in both the lungs, and it was difficult to differentiate them from lung metastasis of pancreatic cancer. Therefore, he underwent neoadjuvant chemoradiotherapy $(50.4 \mathrm{~Gy} / 28 \mathrm{Fr}$ radiotherapy, and 2 cycles of chemotherapy: $600 \mathrm{mg} / \mathrm{m}^{2}$ GEM on days 8 and 22 , and $60 \mathrm{mg} / \mathrm{m}^{2} \mathrm{TS}-1$ on days $1-21$ ). After neoadjuvant chemoradiotherapy, his tumor marker levels were still within the normal ranges. The pancreatic tumor slightly shrunk, and small lung nodules showed no change. We suspected the lung nodules were not metastasis of the pancreatic cancer; therefore, he underwent radical antegrade
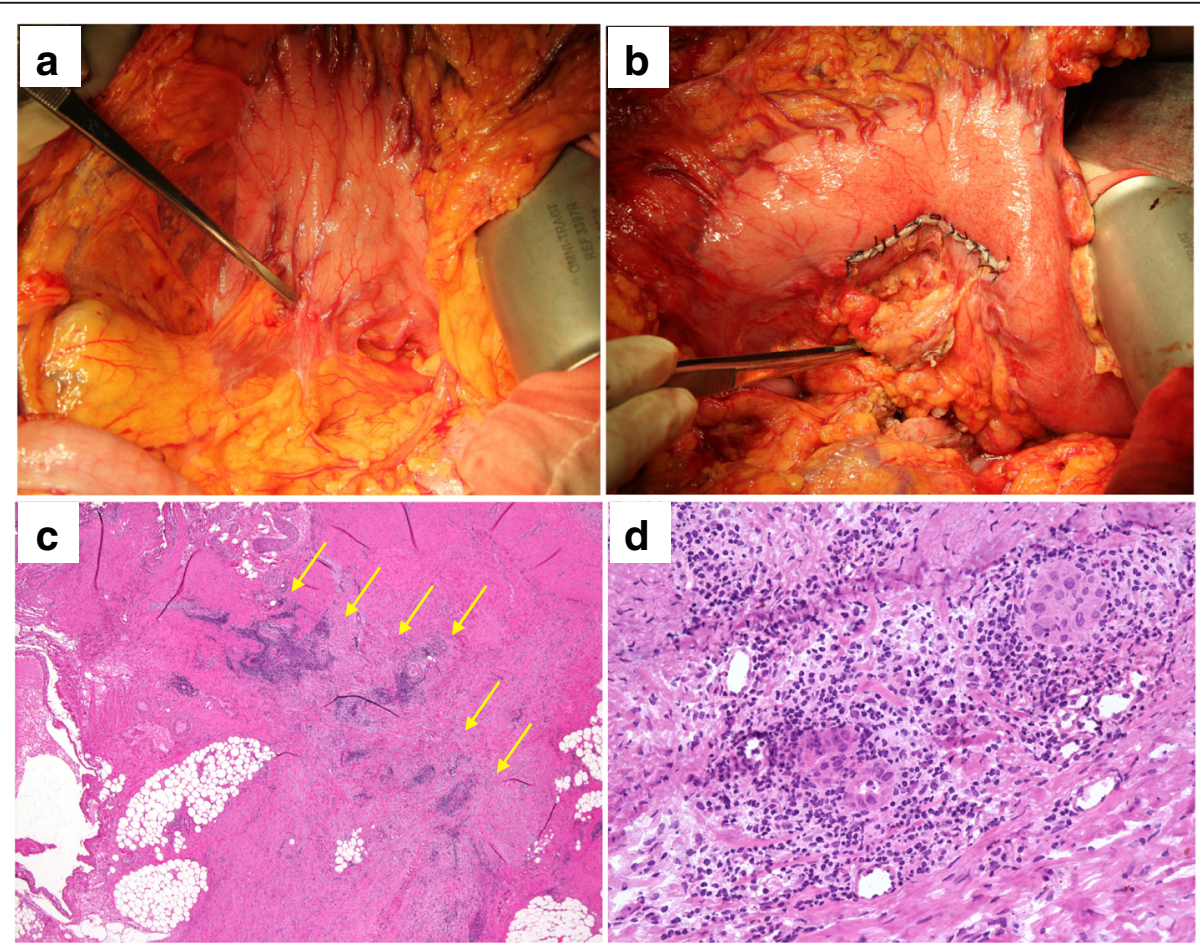

Fig. 2 a Intraoperative findings for case 1. A small hard mass was detected in the posterior gastric wall, as indicated by the forceps. b Partial resection of the posterior gastric wall was performed. c Pathological findings. The specimen from the partially resected stomach showed that an adenocarcinoma was distributed linearly in the gastric muscle layer (arrow) (hematoxylin and eosin staining, loupe image). d Pathological findings. The findings of the gastric tumor were similar to those of the primary pancreatic cancer, indicating that gastric tumor was needle tract seeding from pancreatic cancer (hematoxylin and eosin staining) 


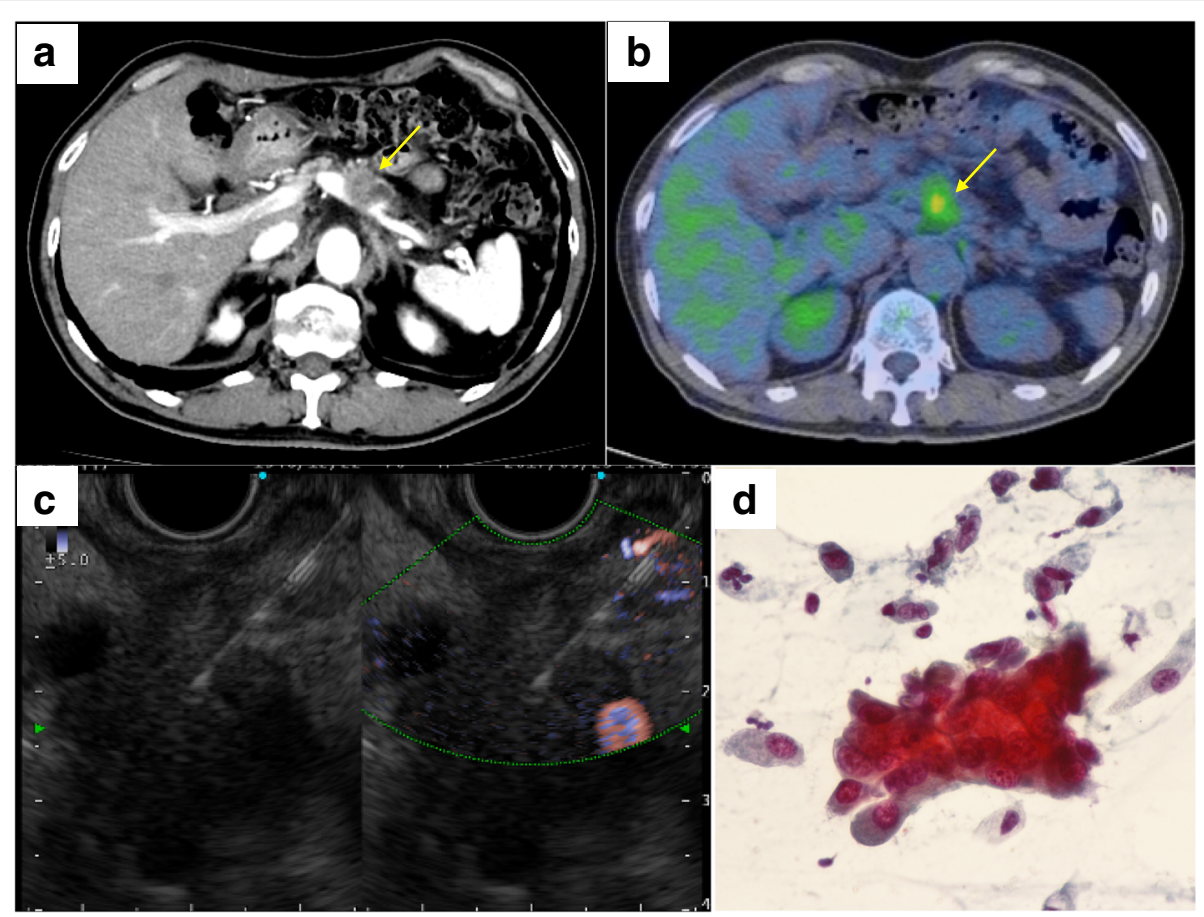

Fig. 3 a Dynamic-enhanced $\subset$ (portal phase) for case 2. A 15-mm hypovascular tumor in the pancreatic body (arrow). b Positron emission tomography- $\subset T$ (PET-CT) findings. Abnormal accumulation of fluorine-18-deoxyglucose (standardized uptake value of 3.74) can be observed in the pancreatic body (arrow). c EUS-FNA findings. EUS-FNA was performed for the pancreatic tumor (1 puncture using $22 \mathrm{G}$, needle) via the trans-gastric approach, without any complications. d Pathological findings. EUS-FNA revealed an adenocarcinoma (Papanicolaou staining)

modular pancreatosplenectomy procedure posterior (RAMPs posterior) [21]. During surgery, we noticed a small hard mass in the posterior gastric wall (Fig. 4a), for which we performed partial gastrectomy (Fig. 4b). The resected specimen was diagnosed as needle tract seeding following EUS-FNA (Fig. 4c, d). The time from EUS-FNA to the detection of the gastric wall metastasis due to needle tract seeding was 113 days. At the end of the surgery, a small nodule was found in the mesenterium of the small intestine. We resected it, and on pathological examination, it was diagnosed as peritoneal dissemination. Pathological findings resulted in a diagnosis of invasive ductal carcinoma, pT2, pN0, and pM1 pStageIV (UICC). He received chemotherapy with only TS-1, as GEM could not be used owing to allergic reactions observed during neoadjuvant chemoradiotherapy. His condition is stable even after 18 months after surgery at the time of writing.

\section{Discussion and conclusions}

It has been reported that EUS-FNA for pancreatic tumor has pooled sensitivity and specificity, $92 \%$ and $96 \%$, respectively [1]. The main complications of EUS-FNA for pancreas tumor is bleeding, pancreatitis, post-procedural pain, and so on, but the incidence rate is as low as 1.03\%; therefore, it is considered a safe procedure [2]. Moreover, there was no significant difference in prognosis even when EUS-FNA was performed on the pancreatic body and tail cancer before surgery, and EUS-FNA is now an essential examination for the diagnosis of pancreatic tumors $[22,23]$. However, in these reports, the type, stage, and resectability of pancreatic tumors were different and EUS-FNA has a risk of peritoneal dissemination, although its diagnosis due to FNA is difficult because pancreatic cancer itself often results in the development of peritoneal dissemination. Hence, the adverse effects of EUS-FNA may be ambiguous. In the future, the oncological safety of EUS-FNA should be reconsidered in limiting patients who undergo this procedure.

The first case of needle tract seeding after EUS-FNA in a patient with invasive ductal carcinoma derived from intraductal papillary mucinous neoplasm (IPMN) was first reported in 2003 [5], then in 2005, needle tract seeding after EUS-FNA was reported in a patient with a common type of pancreatic adenocarcinoma [6].In a search of the PubMed database and Ichushi (Japanese database) using the search term "[(endoscopic ultrasound fine-needle aspiration) OR (EUS-FNA) AND (pancreatic cancer) OR (pancreatic adenocarcinoma) AND (needle tract seeding) OR (seeding)]," till date, only 18 cases (17 reports) of needle tract seeding associated with EUS-FNA for pancreatic cancer have been reported, including invasive ductal carcinoma derived from intraductal papillary mucinous neoplasm (Table 1) [4-20]. Regarding the tumor site, all the tumors were located in the 


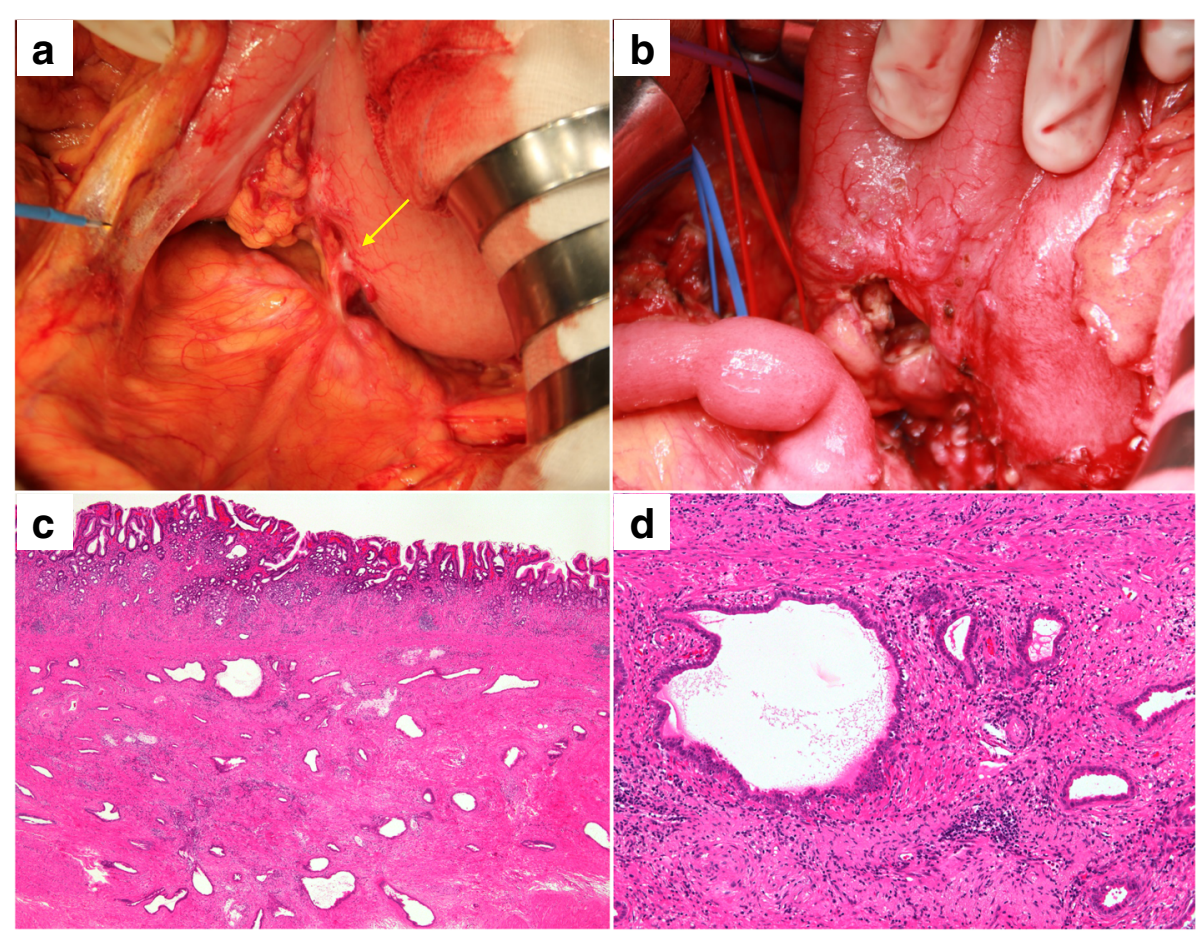

Fig. 4 a Intraoperative findings for case 2. A small hard mass was detected in the posterior gastric wall (arrow). b Partial resection of the posterior gastric wall was performed. c Pathological findings. Many abnormal luminal structures (adenocarcinoma) were confirmed in the resected gastric muscle layer (hematoxylin and eosin staining, loupe image). $\mathbf{d}$ Pathological findings. The findings of gastric tumor were similar to those of the primary pancreatic cancer, indicating that gastric tumor was a recurrence due to needle tract seeding from pancreatic cancer (hematoxylin and eosin staining)

pancreatic body or pancreatic tail, except for a case with pancreatic head cancer who did not undergo surgery, and two cases where there was no description. This was probably because the puncture route is included in the resection range for pancreatic head cancer. In 3 of 18 cases, including our 2 cases, needle tract seeding was detected during surgery. Therefore, intraoperative assessment for gastric wall metastasis is important as well as postoperative assessment, and if surgeon suspects gastric wall metastasis intraoperatively, partial gastrectomy should be performed without hesitation. In these reported cases, the median period until the gastric wall metastasis after EUSFNA is 21 months, but it occurred only 10 days in the shortest case [5]. As shown in our case 2, needle tract seeding after EUS-FNA cannot be controlled even after chemoradiotherapy.

According to NCCN guidelines [24] and clinical practice guidelines for pancreatic cancer 2016 from the Japanese Pancreas Society guidelines [25], the treatment policy of pancreatic cancer varies according to the tumor resectability; surgery is the first treatment choice for resectable pancreatic cancer. For borderline resectable pancreatic cancer, it is a dominant opinion that neoadjuvant chemoradiotherapy is known to improve the prognosis, and for unresectable cases, chemotherapy is chosen. If we choose to perform chemotherapy for pancreatic cancer including preoperative treatment, it is necessary to differentiate it from other pancreatic tumors via EUS-FNA. However, whether EUS-FNA should be performed for all pancreatic tumors is controversial. Depending on the resectability and the localization of the tumor, it is necessary to consider the indications of EUS-FNA separately. For resectable pancreatic cancer that does not conflict with pancreatic cancer on the imaging studies, there may be a choice not to puncture the tumor. When EUS-FNA is performed for pancreatic body or tail cancer which is not included in the resection range, we should be aware of the risk of developing needle tract seeding in the gastric wall. In order to avoid needle tract seeding, biopsy needle with a covering sheath should be used [26]. Although our institution had already used a biopsy needle with a covering sheath, needle tract seeding unfortunately developed in these two cases. Therefore, the other factors such as technical problem should be considered. To prevent needle tract seeding as much as possible, we recommend to avoid unnecessary EUS-FNA for resectable pancreatic body or tail cancer, when up-front surgery is planned. Actually, when we consider the cost of EUS-FNA and the selection of operative procedure for the patients in whom upfront surgery is planned, EUS-FNA has few benefits because EUS-FNA by itself does not influence the selection 


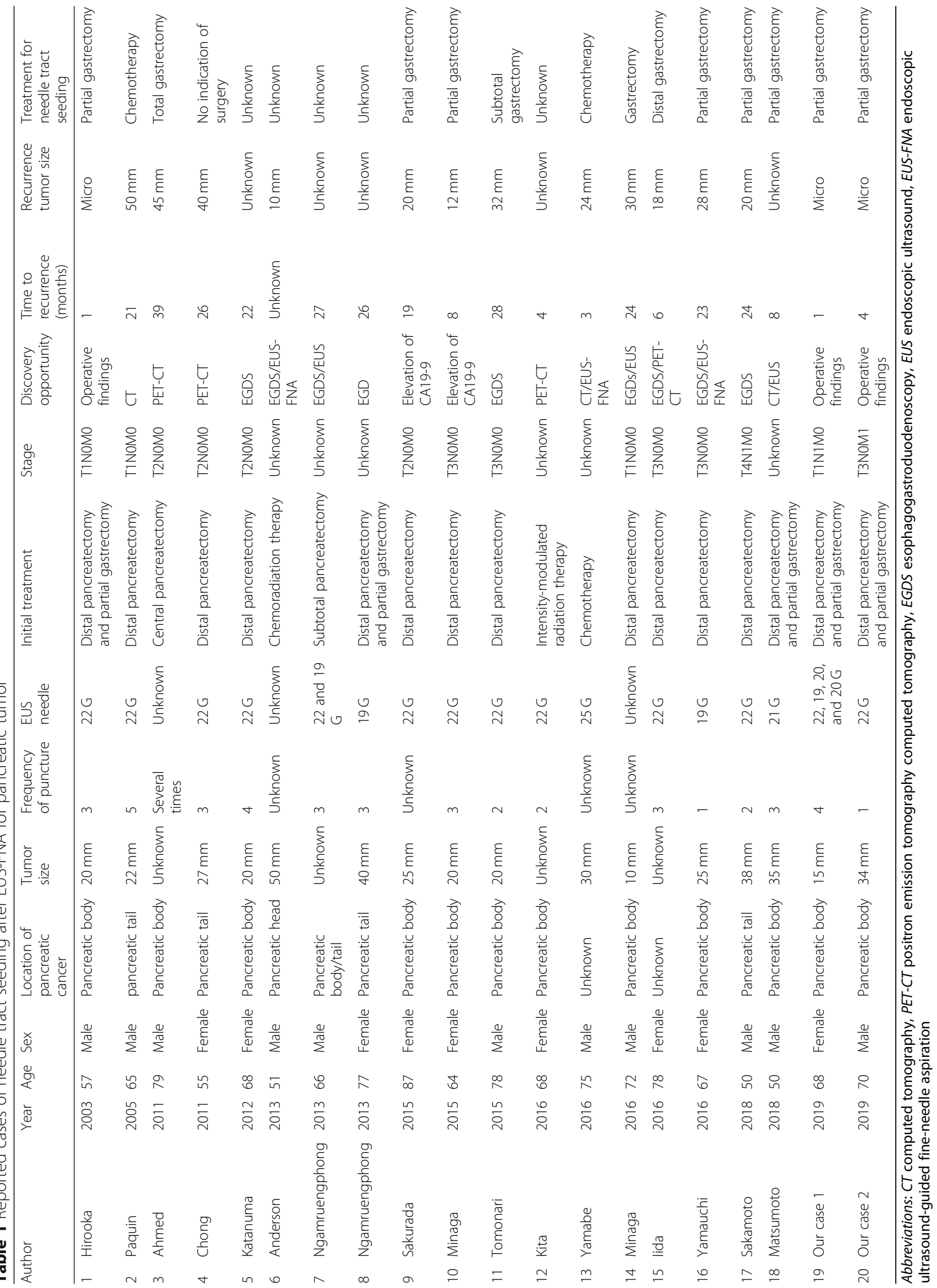


of the operative procedure and is costful. If EUS-FNA is performed, intraoperative and postoperative assessment is essential for gastric wall metastasis due to needle tract seeding. According to the report by Yamauchi et al., if gastric wall metastasis due to needle tract seeding is detected early, partial gastrectomy can control the disease [4]. However, if the finding of gastric wall metastasis due to needle tract seeding is delayed, there is a report that lymph node metastasis has occurred [7]. In addition, there is a report of recurrence after partial gastrectomy for gastric wall metastasis due to needle tract seeding [18]; hence, post-operative assessment is important.

In conclusion, although EUS-FNA is a useful diagnostic tool, it may cause peritoneal dissemination and needle tract seeding at the puncture site. Therefore, physicians should decide its indication, especially for resectable pancreatic body or tail cancer, by taking the consideration of merit and demerit of EUS-FNA for each case.

\section{Acknowledgements}

We are grateful to Shuji Isaji, MD, PhD, Department of Hepatobiliary Pancreatic and Transplant Surgery, Mie University Graduate School of Medicine, for revising this article.

\section{Authors' contributions}

All authors were involved in the preparation of this manuscript. TM wrote the manuscript. KN, HY, ST, YN, and MO performed the endoscopic examination. TM, HN, YS, YH, KT, and MS performed the surgery. KK performed the pathological diagnosis. MS and SI revised the manuscript. All authors read and approved the final manuscript.

\section{Funding}

All authors do not have any funding.

\section{Availability of data and materials}

The datasets obtained during the current study are available from the corresponding author on reasonable request.

\section{Ethics approval and consent to participate}

Not applicable.

\section{Consent for publication}

We obtained patients' consent for publication.

\section{Competing interests}

The authors declare that they have no competing interests.

\section{Author details}

'Department of Surgery, Matsusaka Municipal Hospital, 1550, Tonomachi, Matsusaka, Mie, Japan. ${ }^{2}$ Department of Gastroenterology, Matsusaka Municipal Hospital, 1550, Tonomachi, Matsusaka, Mie, Japan. ${ }^{3}$ Department of Pathology, Matsusaka Municipal Hospital, 1550, Tonomachi, Matsusaka, Mie, Japan. ${ }^{4}$ Department of Hepatobiliary Pancreatic and Transplant Surgery, Graduate School of Medicine, Mie University, Tsu, Japan.

Received: 24 April 2019 Accepted: 29 July 2019

Published online: 05 August 2019

\section{References}

1. Hewitt MJ, McPhail MJW, Possamai L, Dhar A, Vlavianos P, Monahan KJ. EUSguided FNA for diagnosis of solid pancreatic neoplasms: a meta-analysis. Gastrointest Endosc. 2012;75:319-31.

2. Wang KX, Ben QW, Jin ZD, Du YQ, Zou DW, Liao Z, et al. Assessment of morbidity and mortality associated with EUS-guided FNA: a systematic review. Gastrointest Endosc. 2011;73:283-90.
3. Micames C, Jowell PS, White R, Paulson E, Nelson R, Morse M, et al. Lower frequency of peritoneal carcinomatosis in patients with pancreatic cancer diagnosed by EUS-guided FNA vs. percutaneous FNA. Gastrointest Endosc. 2003:58:690-5.

4. Yamauchi J, Kobayashi S, Miyazaki K, Ajiki T, Tsuchihara K, Ishiyama S. A case of curative resection of needle tract seeding after EUS-guided fine needle aspiration for pancreatic body cancer. J Jpn Surg Assoc. 2016;77(12):2994-9.

5. Hirooka Y, Goto H, Itoh A, Hashimoto S, Niwa K, Ishikawa H, et al. Case of intraductal papillary mucinous tumor in which endosonograpy-guided fineneedle aspiration biopsy caused dissemination. J Gastroenterology Hepatol. 2003;18:1323-4

6. Paquin SC, Gariépy G, Lepanto L, Bourdages R, Raymond G, Sahai AV. A first report of tumor seeding because of EUS-guided FNA of a pancreatic adenocarcinoma. Gastrointest Endosc. 2005;61:610-1.

7. Ahmed K, Sussman JJ, Wang J, Schmulewitz N. A case of EUS-guided FNArelated pancreatic cancer metastasis to the stomach. Gastrointest Endosc. 2011;74:231-3.

8. Chong A, Venugopal K, Segarajasingam D, Lisewski D. Tumor seeding after EUSguided FNA of pancreatic tail neoplasia. Gastrointest Endosc. 2011;74:933-5.

9. Katanuma A, Maguchi H, Hashigo S, Kaneko M, Kin T, Yane K, et al. Tumor seeding after endoscopic ultrasound-guided fine-needle aspiration of cancer in the body of the pancreas. Endoscopy. 2012;44:E160-1.

10. Anderson B, Singh J, Jafri SF. Tumor seeding following endoscopic ultrasonography-guided fine-needle aspiration of a celiac lymph node. Dig Endosc. 2013;25:344-5.

11. Ngamruengphong S, Xu C, Woodward TA, Raimondo M, Stauffer JA, Asbun $\mathrm{HJ}$, et al. Risk of gastric or peritoneal recurrence, and long-term outcomes, following pancreatic cancer resection with preoperative endosonographically guided fine needle aspiration. Endoscopy. 2013;45: 619-26.

12. Sakurada A, Hayashi T, Ono M, Ishiwatari H, Ogino J, Kimura Y, et al. A case of curatively resected gastric wall implantation of pancreatic cancer caused by endoscopic ultrasound-guided fine-needle aspiration. Endoscopy. 2015; 47:198-9.

13. Minaga K, Kitano M, Yamashita Y. Surgically resected needle tract seeding following endoscopic ultrasound-guided fine-needle aspiration in pancreatic cancer. J Hepatobiliary Pancreat Sci. 2015;22:708-9.

14. Tomonari A, Katanuma A, Matsumori T, Yamazaki H, Sano I, Minami R, et al. Resected tumor seeding in stomach wall due to endoscopic ultrasonography-guided fine needle aspiration of pancreatic adenocarcinoma. World J Gastroenterol. 2015;21:8458-61.

15. Kita E, Yamaguchi T, Sudo K. A case of needle tract seeding after EUSguided FNA in pancreatic cancer, detected by serial positron emission tomography/CT. Gastrointest Endosc. 2016;84:869-70.

16. Yamabe A, Irisawa A, Shibukawa G, Hoshi K, Fujisawa M, Igarashi R, et al. Rare condition of needle tract seeding after EUS-guided FNA for intraductal papillary mucinous carcinoma. Endosc Int Open. 2016;4:E756-8.

17. Minaga K, Kitano M, Enoki E, Kashida H, Kudo M. Needle-tract seeding on the proximal gastric wall after EUS-guided fine-needle aspiration of a pancreatic mass. Am J Gastroenterol. 2016;111:1515.

18. lida T, Adachi T, Ohe Y, Nakagaki S, Yabana T, Kondo Y, et al. Re-recurrence after distal gastrectomy for recurrence caused by needle tract seeding during endoscopic ultrasound-guided fine-needle aspiration of a pancreatic adenocarcinoma. Endoscopy. 2016;48:E304-5.

19. Sakamoto U, Fukuba N, Ishihara S, Sumi S, Okada M, Sonoyama H, et al. Postoperative recurrence from tract seeding after use of EUS-FNA for preoperative diagnosis of cancer in pancreatic tail. Clin J Gastroenterol. 2018;11:200-5.

20. Matsumoto K, Kato H, Tanaka N, Okada H. Preoperative detection of tumor seeding after endoscopic ultrasonography-guided fine needle aspiration for pancreatic cancer. Intern Med. 2018;57:1797-8.

21. Strasberg SM, Drebin JA, Linehan D. Radical antegrade modular pancreatosplenectomy. Surgery. 2003;133:521-7.

22. Beane JD, House MG, Coté GA, DeWitt JM, Al-Haddad M, LeBlanc JK, et al. Outcomes after preoperative endoscopic ultrasonography and biopsy in patients undergoing distal pancreatectomy. Surgery. 2011;150:844-53.

23. Ngamruengphong S, Swanson KM, Shah ND, Wallace MB. Preoperative endoscopic ultrasound-guided fine needle aspiration does not impair survival of patients with resected pancreatic cancer. Gut. 2015;64:1105-10.

24. Tempero MA, Malafa MP, Al-Hawary M, Asbun H, Bain A, Behrman SW, et al. Pancreatic adenocarcinoma, version 2. 2017, NCCN Clinical Practice 
Guidelines in Oncology. J Nati Compr Canc Netw. 2017;15:1028-61. https:// doi.org/10.6004/jncen.2017.0131.

25. Yamaguchi K, Okusaka T, Shimizu K, Furuse J, Hanada K, Shimosegawa T, et al. Clinical practice guidelines for pancreatic cancer 2016 from the Japanese Pancreas Society: a synopsis. Pancreas. 2017;46:595-604. https://doi.org/10.1 097/MPA.0000000000000816.

26. Tyagi R, Dey P. Needle tract seeding: an avoidable complication. Diagnostic Cytopathol. 2014;42:636-40.

\section{Publisher's Note}

Springer Nature remains neutral with regard to jurisdictional claims in published maps and institutional affiliations.

Ready to submit your research? Choose BMC and benefit from:

- fast, convenient online submission

- thorough peer review by experienced researchers in your field

- rapid publication on acceptance

- support for research data, including large and complex data types

- gold Open Access which fosters wider collaboration and increased citations

- maximum visibility for your research: over $100 \mathrm{M}$ website views per year

At $\mathrm{BMC}$, research is always in progress.

Learn more biomedcentral.com/submissions 